(1)

CrossMark

\title{
Is high-dose glucocorticoid beneficial in COVID-19?
}

To the Editor:

We read with interest the article by EDALATIFARD et al. [1], and we congratulate them for performing a randomised controlled trial amidst the ongoing pandemic. We have a few concerns regarding the methods and interpretation of the current study. While the intervention group's mortality is low, the mortality in the control group is disproportionately high for the severity of illness (acute respiratory distress syndrome (ARDS) not present, patients not intubated). The mortality in ARDS due to coronavirus disease 2019 (COVID-19) among mechanically ventilated patients is $50 \%$ or less, and it has improved over time from $42 \%$ to $25 \%[2,3]$. The mortality in the current study's control arm $(43 \%)$ was worse than the mortality in the control arm of the RECOVERY trial (26\%) [4]. The survival difference between the two study groups may be due to the better supportive care provided to the intervention arm due to the trial's unblinded nature. Also, the justification provided for the sample size is inadequate. The authors have cited studies using methylprednisolone in asthma, COPD and pre-operative patients as references for the sample size calculation.

The primary outcome chosen was clinical improvement, defined as "Borg score $>3$, improved dyspnoea, no fever for $72 \mathrm{~h}, \mathrm{~S}_{\mathrm{pO}_{2}}>93 \%$, tolerated oral regime, normal urinary output, and reduced CRP level without any treatment side-effects". Most of these are subjective parameters. The quick defervescence and a sense of well-being intuitively explain the improvement in the subjective parameters observed in the study group. The choice of Borg scale and subjective sensation of dyspnoea for acute respiratory failure, instead of more reliable and objective scores, such as arterial oxygen tension/inspiratory oxygen fraction ratio, APACHE-II or the SOFA score, raises concerns regarding the interpretation of the trial.

Finally, diabetes mellitus was the most common comorbidity in the study. However, more patients in the control group had diabetes mellitus, which spuriously decreased the observed adverse events in the intervention arm. Such high doses of glucocorticoids can predispose the patients to devastating complications such as COVID-19-associated pulmonary aspergillosis and COVID-19-associated mucormycosis. We believe that more extensive research is required before high-dose glucocorticoids can be recommended for treating severe COVID-19 patients.

@ERSpublications

High-dose glucocorticoids are not currently recommended for treating severe COVID-19 pneumonia https://bit.ly/39VQ3O8

Cite this article as: Muthu V, Sehgal IS, Prasad KT, et al. Is high-dose glucocorticoid beneficial in COVID-19? Eur Respir J 2021; 57: 2100065 [https://doi.org/10.1183/13993003.00065-2021].

Valliappan Muthu, Inderpaul Singh Sehgal (1), Kuruswamy Thurai Prasad and Ritesh Agarwal

Dept of Pulmonary Medicine, Postgraduate Institute of Medical Education and Research (PGIMER), Chandigarh, India.

Correspondence: Ritesh Agarwal, Dept of Pulmonary Medicine, Postgraduate Institute of Medical Education and Research, Sector 12, Chandigarh 160012, India. E-mail: agarwal.ritesh@outlook.in

Received: 9 Jan 2021 | Accepted: 17 Jan 2021

Conflict of interest: None declared.

\section{References}

1 Edalatifard M, Akhtari M, Salehi M, et al. Intravenous methylprednisolone pulse as a treatment for hospitalised severe COVID-19 patients: results from a randomised controlled clinical trial. Eur Respir J 2020; 56: 2002808.

2 COVID-ICU Group on behalf of the REVA Network and the COVID-ICU Investigators. Clinical characteristics and day-90 outcomes of 4244 critically ill adults with COVID-19: a prospective cohort study. Intensive Care Med 2021; 47: 60-73.

3 Lim ZJ, Subramaniam A, Ponnapa Reddy M, et al. Case fatality rates for patients with COVID-19 requiring invasive mechanical ventilation. A meta-analysis. Am J Respir Crit Care Med 2021; 203: 54-66. 
4 RECOVERY Collaborative Group, Horby P, Lim WS, et al. Dexamethasone in hospitalized patients with Covid-19. N Engl J Med 2021; 384: 693-704.

Copyright (๑) The authors 2021.

This version is distributed under the terms of the Creative Commons Attribution Non-Commercial Licence 4.0. For commercial reproduction rights and permissions contact permissionsdersnet.org 